\title{
Ein Beitrag zur Biographie, insbesondere der letzten Lebensjahre, von Emil Abderhalden (1877-1950)*
}

Von Werner Sackmann

Einer der letzten Forscher und Lehrer, die Physiologie und physiologische Chemie noch in gleichem Maße überblickten und unterrichteten, war der Deutschschweizer Emil Abderhalden; Deutsch-Schweizer nicht nur, weil er aus der deutschsprachigen Schweiz stammte, sondern auch deshalb, weil er sein aktives Forscherleben nahezu ausschließlich in Deutschland zubrachte und sich mindestens ebensosehr als Deutscher verstand.

Aus der Endzeit des Zweiten Weltkrieges und den ersten Nachkriegsjahren hat Abderhalden ein Tagebuch hinterlassen, das eine Durchsicht wahrhaft verdient. Seine vollständige Wiedergabe allerdings wäre teils uninteressant, teils unangebracht. Einmal verzeichnet es großenteils nur Absender und Adressaten der täglich eintreffenden bzw. abgehenden Briefpost. Daraus ein Personenverzeichnis zu erstellen und so die mitmenschliche Umwelt Abderhaldens zu charakterisieren, ist zwar vorgesehen, würde jedoch den hier zur Verfügung stehenden Raum sprengen. Zum anderen können die wenigen zusammenhängenden Gedanken und Erinnerungen, die sich vorfinden, aus Gründen des Personenschutzes heutigentages noch nicht lückenlos wiedergegeben werden.

Außerdem enthält das Tagebuch aber noch eine ansehnliche Zahl von stichwortartigen Eintragungen und Angaben darüber, wie Abderhalden von Deutschland Abschied nehmen mußte und wie er in der Schweiz Fuß faßte. Diese unmittelbaren biographischen Quellen sollen hiemit erstmals benützt und so das Tagebuch kurz vorgestellt werden. Unberücksichtigt bleiben dabei anderweitige Hinweise, z. B. Sorgen gesundheitlicher Art oder das Schicksal von Angehörigen betreffend.

Von den nicht minder zahlreichen Anhaltspunkten, die das Tagebuch zu Abderhaldens schriftstellerischer Leistung liefert, wird nur das fortlaufend numerierte Verzeichnis der fertiggestellten wissenschaftlichen Publikationen benützt, das anfänglich mit den Tagesnotizen seitenweise alterniert. Dieses gab den Anstoß, nach weiteren Veröffentlichungen aus Abderhaldens

* Herrn Prof. Dr. Heinrich Buess zum 70. Geburtstag dankbar zugeeignet. 
Feder Umschau zu halten und - als Abschluß des vorliegenden Beitrages ein Schriftenverzeichnis für die letzten Lebensjahre zu erstellen.

\section{Biographische Vorbemerkungen}

Über Abderhaldens Person und Lebenswerk besteht eine recht umfangreiche Literatur, auf die hier nicht eingegangen wird. Zum biographischen Hintergrund mögen die folgenden Hinweise genügen.

Aus altem Toggenburger Geschlecht stammend, wuchs Abderhalden als Lehrerssohn in Basel auf, wo er Mittelschule und Universität besuchte, Arzt wurde und zum Dr.med. promovierte ${ }^{1}$. Seine maßgeblichen Lehrer und Förderer waren der Physiologe Gustav von Bunge (1844-1920) ${ }^{2,3,4}$ in Basel, später der Eiweißchemiker Emil Fischer (1852-1919) ${ }^{5,6,7}$ in Berlin, wo Abderhalden sich 1904 habilitierte und 1908 Professor an der Tierärztlichen Hochschule wurde. 1911 folgte er einem Ruf als Ordinarius für Physiologie und physiologische Chemie an die Universität Halle a.d. Saale, wo er während 34 Jahren eine überaus reiche Wirksamkeit entfaltete. Seine Verdienste, die hier nicht einzeln gewürdigt werden können, liegen in den Bereichen der Proteinchemie, der Eiweißernährung, der Vitaminforschung, der Endokrinologie und der Enzymforschung. Sein Name bleibt in besonderer Weise verbunden mit den sog. Abwehrfermenten ${ }^{8}$, ferner mit dem nach ihm und Guido Fanconi benannten Syndrom der Cystindiathese ${ }^{9,10}$.

\section{Zum Tagebuch}

Anregung zur Führung eines Tagebuchs war vermutlich die kriegsbedingte Bedrohung Deutschlands und die Vorahnung umwälzender Ereignisse. Die ersten Notizen (8. November 1943) * betreffen die Evakuation von Besitztümern der Leopoldinischen Akademie, deren Präsident Abderhalden seit 1931 war, und die Verbringung dieses Materials sowie privater Effekten in den stillgelegten Schacht eines Kalibergwerkes. Auch werden die ersten Bombenangriffe vom 7. und 16. Juli 1944 erwähnt; weitere sind am 12. und 31. März und am 6. April 1945 notiert. Dem deutschen Verteidigungsangriff an der Ostfront vom 16. Dezember 1944 folgt am 12. Januar 1945 die

* Kalenderdaten und Zeitangaben sind alle dem Tagebuch entnommen, ebenso einige Seitenzahlen. 
russische Großoffensive, die zusammen mit dem Vormarsch der westlichen Alliierten das Ende herbeiführt. In diesen Tagen, am 23. Dezember 1944, blickt Abderhalden in aller Stille auf vierzig Jahre akademischer Lehrtätigkeit zurück.

Am 16. April 1945 wird Halle von amerikanischen Truppen eingenommen. Da die Siegermächte hernach jedoch eine weiter westlich verlaufende Demarkationslinie vereinbaren, müssen die Amerikaner das Gebiet kurzfristig wieder räumen. Sie wollen aber den Sowjets nicht alles so übergeben, wie sie es angetreten haben. Sie transportieren daher nicht nur allerhand Vorräte, Waren, Einrichtungen und Maschinen ab; es kommt auch zur Deportation ganzer Bevölkerungsteile. Alle namhaften Vertreter aus Industrie, Handel, Wissenschaft und Kultur sollen nämlich dem Westen erhalten bleiben.

Am 21. Juni 1945 erhält Abderhalden Befehl, sich mit seiner Familie, d.h. mit Gattin, Sohn, Schwiegertochter, zwei verheirateten Töchtern und einem Enkelkind, zum Abtransport einzufinden. Am 24.Juni treten 730 Hallenser Bürger eine mühselige, entbehrungsreiche Güterzugreise an und gelangen nach fünf langen Tagen über Nordhausen, Erfurt, Bebra und Kassel nach Hanau bei Frankfurt. Im Raume Darmstadt werden die Deportierten auf Bauerndörfer verteilt und harren der neuen Wohnstätten und Arbeitsplätze, die ihnen versprochen sind. Es folgen Monate des Müßigganges, des Hungers und der Zermürbung.

Damit hat Abderhaldens Lebenswerk in Halle ein jähes Ende gefunden. «Ich verlor alles: Vermögen, Haus, Institut, Akademie!» (S.48). Noch lange wird jede Verbindung mit Halle gesucht und jede Nachricht von dort geprüft, ob sie eine Rückkehr ratsam und möglich erscheinen lasse. Schließlich aber obsiegt der nicht minder naheliegende Entschluß, die Rettung in der vom Kriege verschonten Schweiz, dem Lande der Väter, zu suchen und dort noch einmal eine Existenz aufzubauen.

Nach langem Hin und Her und enormen Schwierigkeiten gelingt es schließlich, mit einem Lastwagentransport des Schweizerischen Roten Kreuzes am 7.September 1945 nach Basel einzureisen. Dort trifft man lang entbehrte Freunde wieder, die fürs erste weiterhelfen. Dann finden die Eltern Aufnahme bei ihrer Tochter Ruth, die in der Schweiz studiert hat und in St. Gallen als Pfarrerin tätig ist. Die Woche hindurch weilt Abderhalden gewöhnlich in Zürich und logiert an der Zürichbergstraße bei dem hilfreichen Freund Eduard Rübel (1876-1960), Professor für Geobotanik an der Eidgenössischen Technischen Hochschule. Auf den 1. April 1946 kann die Familie sodann an der Restelbergstraße 56 in Zürich 7 (Fluntern) eine eigene 
Mietwohnung beziehen. - Nach Tagen der Erholung in Basel und St. Gallen begibt Abderhalden sich erstmals am 26. September 1945 nach Zürich und stellt die ersten persönlichen Kontakte mit der Universität her. Er trifft sich mit seinem Fachkollegen, dem Physiologen Walter Rudolf Heß (1881-1973); dem Dekan der medizinischen Fakultät, dem Radiologen Hans Rudolf Schinz (1891-1966), sowie mit dem kantonalen Erziehungsdirektor, Regierungsrat Robert Briner (1885-1960). Während im Verkehr mit Heß eher Zurückhaltung geübt wird, sind Schinz und vor allem Briner dem Bittsteller sehr gewogen. Für das Wintersemester 1945/46 sieht man zunächst eine Gastprofessur vor.

Am 1.Oktober stattet Abderhalden dem interimistischen Direktor des Physiologisch-chemischen Institutes, Karl Bernhard, seinen Antrittsbesuch ab. Bernhard ist seit kurzem Titularprofessor und hat soeben die Institutsleitung übernommen, nachdem der bisherige Lehrstuhlinhaber, Bonifaz Flaschenträger (1894-1959), seines Postens enthoben worden war als Quittung für nationalsozialistische Haltung während des Krieges. Für Bernhard ist das Erscheinen und die Aufnahme eines Hausgenossen vom Ruf und Format Abderhaldens gewiß eine delikate Angelegenheit.

Am 6. Oktober 1945 wird die Ernennung Abderhaldens zum Extraordinarius beschlossen, und schon am 9. Oktober hält er, durch den Dekan eingeführt, seine erste Vorlesung. Bald überträgt ihm die Hochschulkommission auch die Institutsleitung und das Ordinariat in physiologischer Chemie. Diese Ernennung datiert vom 25. Januar 1946, und die Übernahme der Ämter erfolgt am 15. April.

Am 1. Juni 1946 wird Abderhalden brieflich als Präsident der Leopoldinischen Akademie in Halle bestätigt. Einem späteren Tagebucheintrag zufolge soll er 1948 von Theodor Brugsch (1878-1963) in diesem Amte abgelöst worden sein. Dafür war nirgends sonst eine Bestätigung zu finden. Den Annalen der Akademie zufolge ${ }^{11}$ ging das Präsidium vielmehr nach Abderhaldens Tod an den seit 1945 geschäftsführenden Vizepräsidenten Otto Schlüter (1872-1959) über. Mit dem Mediziner Brugsch wie mit dem Geographen Schlüter stand Abderhalden in regem Briefwechsel.

Daß Abderhaldens Einsetzung in Zürich nicht ungeteilten Beifall fand, hatte seinen Grund bestimmt zum Teil in einer allgemeinen, zeitbedingten Abneigung gegen alles Deutsche und von Deutschland Kommende; zudem war es auch keineswegs selbstverständlich, einen Mann von seinem Alter noch als Ordinarius zu berufen. Daß die Satzungen der Universität eine Altersgrenze vorsehen und keine Ausnahme kennen, hätte auch Abderhal- 
den - trotz scheinbar unbegrenzter Schaffensfreude - einsehen müssen. Anstelle dessen beklagt er sich bitter, schon wieder Amt und Arbeitsstätte zu verlieren, als er nach Vollendung seines 70.Altersjahres (9. März 1947) emeritiert und sein Ordinariat auf 15. April in die übliche Honorarprofessur umgewandelt wird. Schon seit November 1946 liefen Verhandlungen mit Franz Leuthardt in Genf, und seit dem 15. Dezember war dessen Berufung nach Zürich offiziell bekannt.

Am 15.Juli 1947 verzeichnet Abderhalden seine letzte ordentliche Vorlesung im Tagebuch. Auf 1.September hat er das Institut zu räumen, «eine unerhörte Grausamkeit»(S.167), die indessen den akademischen Gepflogenheiten in keiner Weise zuwiderläuft.

Im Wintersemester 1946/47 kann Abderhalden noch für einige Vorlesungen nach Basel verpflichtet werden. Durch das überraschende Ableben von Siegfried Edlbacher (1886-1946) ist dort nämlich die physiologische Chemie verwaist. Bis zum 30. Mai 1947 weilt Abderhalden deshalb jede Woche einen Tag am Vesalianum, um den Unterricht in physiologischer Chemie aufrechtzuerhalten und das Interregnum zwischen Edlbachers Tod und dem Amtsantritt von Karl Bernhard zu überbrücken. Das Zustandekommen dieses Lehrauftrages scheint nicht zuletzt dem Fußball-Club Basel zu verdanken zu sein, an dessen Gründung der junge Abderhalden 1893 maßgeblich beteiligt gewesen war ${ }^{12}$. Abderhalden berichtet nämlich von einem Nachtessen im «Schützenhaus», das der FCB am 20. Februar 1947 zu seinem 70. Geburtstag veranstaltet habe und zu dem auch Regierungsratspräsident Fritz Ebi (1889-1961) und Erziehungsdirektor Carl Miville geladen waren ${ }^{13}$. Im Tagebuch ist daran anschließend ein kurzer Briefwechsel mit Miville belegt.

Als Honorarprofessor hält Abderhalden in Zürich ein Kolleg für Hörer aller Fakultäten über «Biologische Tagesfragen»; doch auch das endet mit einer letzten Vorlesung am 18. Juli 1948. Unter dem 15. Oktober spricht das Tagebuch ein zweites Mal von Entamtung.

Ist Abderhalden über das Ende seiner Universitätslaufbahn nicht eben erbaut, so erfreut er sich anderseits zahlreicher Berufungen und Ehrungen. Im Dezember 1945 z. B. ernennt ihn der Verein abstinenter Schweizer Ärzte, im Februar 1948 die Schweizerische Akademie der medizinischen Wissenschaften und im Mai 1948 die St. Galler Naturforschende Gesellschaft zum Ehrenmitglied. Im Juni 1947 beruft ihn das Eidgenössische Gesundheitsamt in die Subkommission für wissenschaftlichen Erfahrungsaustausch, als deren Präsident er noch im selben Jahr gewählt wird. Im Juli 1947 wird er 
ferner vom Bundesrat in die eidgenössische Kommission zur Bekämpfung des Alkoholismus entsandt.

Wenn auch die Zürcher Hochschule seine Dienste als Ordinarius nicht weiter beanspruchen kann, so fehlt es nicht an akademischen Berufungen nach Deutschland, wo ein akuter Mangel an fähigen und politisch unbelasteten Kandidaten herrscht. Dort übersähe man Altersgrenzen leicht und gerne. Es ergehen Rufe aus Tübingen und Mainz, ja auch aus dem heimatlichen Halle sowie aus Leipzig, wohin Abderhalden am 21. Oktober 1947 seine Absage schickt. Offenbar fühlt er sich für einen nochmaligen Neuanfang doch nicht mehr stark genug; auch will er sich ausdrücklich von seinen Kindern, die sich in der Schweiz niedergelassen haben, nicht wieder trennen, am wenigsten von dem heißgeliebten Enkel, dem Sohn seiner verwitweten jüngsten Tochter (S. 170). Eine besondere Freude und Genugtuung bereitet in dieser Situation das Ehrendoktorat, das ihm die Frankfurter Universität am 18. Mai 1948 aus Anlaß ihrer Hundertjahrfeier verleiht.

\section{Veröffentlichungen aus den letzten Lebensjahren}

Abderhalden war von jeher ein ungemein mitteilsamer und publikationseifriger Wissenschafter. Das in seinem Tagebuch Ende 1943 angefangene Publikationsverzeichnis beginnt mit der Bemerkung: «Bis Nr. 1206 ist alles in einem Verzeichnis aufgenommen» (S.27). Dies letztere muß indessen verlorengegangen sein.

Zum Abschluß dieser Mitteilung wurde versucht, Abderhaldens sämtliche Arbeiten, wissenschaftliche und andere, zusammenzustellen, die er nach 1943 noch verfaßt hat. Eine solche Liste dürfte um so willkommener sein, als die bisher erstellten Gesamtverzeichnisse ${ }^{14,15,16}$ über die Zeit der letzten Kriegswirren und den Lebensabend höchst lückenhaft Auskunft geben.

Das hier vorgelegte Verzeichnis übernimmt einmal die Zitate aus dem Tagebuch und ergänzt sie durch alle weiteren in dem entsprechenden Zeitraum angetroffenen Arbeiten. Von den Zitaten im Tagebuch sind wiederum nur diejenigen mit Abderhaldens persönlicher Autorschaft berücksichtigt; die sonst noch erwähnten Arbeiten, z.B. diejenigen seines Sohnes Rudolf (1910-1965), wurden nicht übernommen.

Herrn Dr. Klaus Vosbeck danke ich bestens für die Überlassung des Tagebuches aus dem Nachlaß Abderhaldens. 


\section{Verzeichnis der späten Schriften Abderhaldens (1944-1950)}

Beginnend mit den Lehrbüchern und Monographien, alsdann geordnet nach den verschiedenen Periodica

Lehrbuch der Physiologie, 6. \& 7. Aufl., Berlin/Wien (Urban \& Schwarzenberg) 1944.

Lehrbuch der Physiologie, 8. \& 9. Aufl., Wien (Urban \& Schwarzenberg) 1946.

Lehrbuch der Physiologie, 10.-12. Aufl., Basel (Schwabe) 1946.

Lehrbuch der physiologischen Chemie, 16.-22. Aufl., Wien (Urban \& Schwarzenberg) 1944.

Lehrbuch der physiologischen Chemie, 23.-25. Aufl., Basel (Schwabe) 1946.

Lehrbuch der physiologischen Chemie, 26. Aufl., Basel (Schwabe) 1948.

Physiologisches Praktikum (I.Physiolog. Chemie), 7. Aufl., Frankfurt (Steinkopff) 1946.

Physiologisches Praktikum (I.Physiolog. Chemie), 8. Aufl., Frankfurt (Steinkopff) 1948.

Physiologisches Praktikum (II.Allg. Physiologie), 6. Aufl., Dresden (Steinkopff) 1944.

Physiologisches Praktikum (II. Allg. Physiologie), 7.Aufl., Frankfurt (Steinkopff) 1949.

Spuren von Stoffen entscheiden über unser Schicksal, 2. Aufl., Basel (Schwabe) 1946.

Die Grundlagen unserer Ernährung, 5. Aufl., Bern (Huber) 1946.

Abwehrfermente (Die Abderhaldensche Reaktion), 7.Aufl., Dresden (Steinkopff) 1946.

Gedanken eines Biologen zur Schaffung einer Völkergemeinschaft und eines dauerhaften Friedens, Zürich (Rascher) 1947.

Mit Georges Mouriquand: Vitamine und Vitamintherapie, Bern (Huber) 1948.

Besondere Leistungen der Nieren im Zellstoffwechselgeschehen, in: Nierenkrankheiten, Teil I, S. 544-550 (Hrsg. Erwin Becher), Jena (Fischer) 1944.

Zum 100. Geburtstag Gustav von Bunges. Basler Nachrichten, Nr. 26 (18. Januar 1944).

Die Lebenskräfte im Blut. Physiologische Ansichten von der Leistung der Blut- und Muskelzellen. Das Reich, No.51 (17. Dezember 1944). (Tagebuch Abderhalden: «Titel nicht von mir!»)

Spuren von Stoffen entscheiden über unser Schicksal, 1. Aufl. Nova Acta Leopoldina N. F. 13 (1944) 379-432.

Beitrag zum Virusproblem. Nova Acta Leopoldina N. F. 13 (1944) 483-487.

Bestand und Tätigkeit der Akademie im Jahre 1943. Nova Acta Leopoldina N. F. 13 (1944) 523-550.

Das Verhalten der A.-R. bei Agonie. Fermentforschung 17 (1944) 297-305.

Über den Ausfall der A.-R. bei Fällen von Pubertas praecox. Fermentforschung 17 (1944) $306-310$.

Weiterer Beitrag zur Spezifität der Abwehrproteinasereaktion (A.-R.) an Hand von jodhaltigen Eiweißstoffen. Fermentforschung 17 (1944) 311-315.

Mit Rudolf Abderhalden: Darstellung und Eigenschaften einer hochgereinigten Abwehrproteinase. Fermentforschung 17 (1944) 344-351.

Die quantitative Erfassung der Abwehrproteinasewirkung. Fermentforschung 17 (1944) 374-386.

Mit Gerd Fabian: Weiterer Beitrag zur Kenntnis der Verwendbarkeit der A.-R. zur Diagnose des Karzinoms. Fermentforschung 17 (1945) 457-490.

Der Einfluß psychischer Erregungen auf den Ausfall der A.-R. Fermentforschung 17 (1945) $527-548$. 
Anwendung der A.R. zur Prüfung der Auswirkung von Verbindungen, die eine bestimmte Wirkung auf den Organismus ausüben: Tyramin, Hordenin, Adrenalin, Ephedrin, Pervitin, Veritol. Fermentforschung 17 (1945) 562-572.

Isolierung von Aminosäuren aus der Bebrütungsflüssigkeit Plazenta plus Abwehrproteinasen aus Schwangerenharn. Fermentforschung 17 (1945) 573-576.

Mit Alexander Parschin: Weitere Untersuchungen über das Verhalten von Diketopiperazinen im tierischen Organismus. Fermentforschung 17 (1945) 577-582. Vgl. Z. Vitamin-, Hormon- \& Fermentforsch.1 (1947) 21-26.

Mit Alexander Parschin: Studien über das Verhalten von Histidinanhydrid, Histidyl-histidin und Karnosin gegenüber Fermenten. Fermentforschung 17 (1945) 591-599. Vgl.Z. Vitamin-, Hormon- \& Fermentforsch.1 (1947) 11-20.

Mit Rudolf Abderhalden und Josef Klumpar: Über die Verwendung der polarographischen Methode zum Nachweis der Wirkung von Proteinasen und insbesondere von Abwehrproteinasen. Fermentforschung 17 (1945) 600-609.

Fortschritte auf dem Gebiete der Abwehrfermentforschung. Forschungen und Fortschritte 21 (1945) 5-7.

Loblied der roten Gläser. Aus Theorie und Praxis. Luftwaffenkurier Ost, Folge 68 (1.Jan. 1945). (Tagebucheintrag Abderhaldens: «Titel nicht von mir!» Der Hinweis ließ sich nicht verifizieren.)

Ein kultureller Völkerbund für Genf. Weltlenkung der Ernährungs- und Gesundheitsprobleme. Solothurner Zeitung, Nr.38 (14.Februar 1946); Der Freisinnige (Wetzikon), Nr.43 (21. Februar 1946); St. Galler Tagblatt, Nr. 100 (28. Februar 1946).

Assoziationen und Dissoziationen in der Organismenwelt. Festschrift Eduard Rübel, S.221234, Zürich (Schultheß) 1946.

Ferdinand Edler von Neureiter, o. Prof. für Gerichtliche Medizin. Dtsch.med. Wschr.71 (1946) 191.

Alloxandiabetes. Dtsch.med.Wschr.71 (1946) $241 \mathrm{f}$.

Verwendbarkeit der Abwehrproteinasereaktion zur Diagnose des Karzinoms. Schweiz. med. Wschr.76 (1946) 47.

Über Abwehrproteinasen. Schweiz.med. Wschr.76 (1946) 546.

Allgemeine Bedeutung reziproken Geschehens in unserem Organismus und sein Wesen. Schweiz. med.Wschr.76(1946) 767.

Ist Cholin ein Vitamin? Schweiz.med.Wschr.77 (1947) 629 (Festschr. Kurt von Neergaard). Thiamin-Avitaminose. Schweiz.med. Wschr. 77 (1947) 689f. (Festschr. Wilhelm Löffler).

Studien über Alloxandiabetes. Schweiz.med.Wschr.77 (1947) 1145 f. (Festschr. Guido Miescher).

Aktuelle Probleme der Ernährung. Med.Klin.42 (1947) 503-505.

Neuer Weg zum Studium der Avitaminosen. Arch.néerl. Physiol. 28 (1947) 373-376 (Festschr.

G. van Rijnberk).

Das Makromolekül Eiweiß. Makromolek. Chemie 1 (1947) 85-88.

Probleme der Ernährung. Der Bogen (Wiesbaden) 2 (1947) $27 \mathrm{f}$.

Das Wasser als physiologischer Faktor. CIBA Zeitschrift 9 (1947) 3912-3918.

Wissenschaftliche Selbstbiographie von Max Planck. Neue Zürcher Zeitung, Nr. 1896 (11. Oktober 1947) und Nr.2036 (18. Oktober 1947).

Nur die unverfälschte Natur kann siegen. Die Freiheit (Lausanne) 55 (1947), Nr. 16. 
Beitrag zum Antialkoholunterricht. Pflugschar (Bern) 41 (1947) 23-25.

Einführung. Z. Vitamin-, Hormon- \& Fermentforsch. 1 (1947) 1-7.

Fragen der Nomenklatur. Z. Vitamin-, Hormon- \& Fermentforsch. 1 (1947) 8-10.

Mit Alexander Parschin: Studien über das Verhalten von Histidinanhydrid, Histidyl-histidin und Karnosin gegenüber Fermenten. Z. Vitamin-, Hormon- \& Fermentforsch.1 (1947) 11-20. Vgl. Fermentforschung 17 (1945) 591-599.

Mit Alexander Parschin: Weitere Untersuchungen über das Verhalten von Diketopiperazinen im tierischen Organismus. Z.Vitamin-, Hormon- \& Fermentforsch.1 (1947) 21-26. Vgl. Fermentforschung 17 (1945) 577-582.

Hervorrufung einer Thiamin-Avitaminose bei Tauben und Ratten mittels Thiaminase. Z. Vitamin-, Hormon- \& Fermentforsch. 1 (1947) 55-63.

Thiaminase. Z. Vitamin-, Hormon- \& Fermentforsch. 1 (1947) 186-190.

Alloxandiabetes. Z. Vitamin-, Hormon- \& Fermentforsch. 1 (1947) 191-198.

Beitrag zum Problem der Alloxan-, Alloxantin-, Ninhydrin- und Ninhydrinharnstoffwirkung auf den tierischen Organismus. Z. Vitamin-, Hormon- \& Fermentforsch. 1 (1947) 241-256.

Lassen sich verschiedenartige Störungen des lymphatischen Systems mittels der A.-R. unterscheiden? Z. Vitamin-, Hormon- \& Fermentforsch. 1 (1947) 289-295.

Studien über den Ausfall der A. R. mit Substrat aus Plazenta, Hormonorganen und bestimmten Anteilen des Nervengewebes in verschiedenen Monaten und Zuständen der Schwangerschaft. Z. Vitamin-, Hormon- \& Fermentforsch. 1 (1948) 318-336.

Aktuelle Probleme der Ernährung. Dtsch.med.Rundsch.(Köln) 1 (1947) 105-107.

Ärzte an die Front. Dtsch.med.Rundsch. (Köln) 1 (1947) $313 \mathrm{f}$.

Medikamentöse Behandlung des Alkoholismus. Dtsch.med.Rundsch. (Köln) 3 (1949) 215 f.

Neuere Ergebnisse auf dem Gebiete der Vitaminforschung. Bull.Schweiz. Akad.med. Wiss. 2 (1947) 331-344.

Wandlungen in der Auffassung des Wesens der Alternsvorgänge. Bull. Schweiz. Akad. med. Wiss. 6 (1950) 102-114.

Gegen den Hunger. Echo. Deutsche Warte in Bayern (Nürnberg) 3 (1948) 15. (Zitat aus dem Tagebuch, das sich nicht verifizieren ließ.)

Naturwissenschaft, Medizin und Weltfrieden. Zürcher Student 26 (1948) 112-116.

Ist die Existenz eines «aminotoxischen Komplexes» (ATK) erwiesen? Dtsch. zahnärztl. Zeitschr.4 (1949) $722 \mathrm{f}$.

Schlußbericht über die Tätigkeit des "Hilfswerkes für das geistige Deutschland». Eigendruck, 28. Februar 1949.

Persönliche Erinnerungen an die Entdeckung der Eiweißsynthese im tierischen Organismus aus freien Aminosäuren und die Erforschung der biologischen Wertigkeit der einzelnen Eiweißbausteine. Ernährg. \& Verpflegung (Berlin) 1 (1949) 71-73; Materia medica, Nordmark 1950.

Einheit der Vitamine, Hormone, Fermente. Volksgesundheitsdienst (Staufen) 1 (1950) 35-38. Vermögen Wissenschaft und Technik die Ernährung der Menschheit sicherzustellen? Atlantis 22 (1950) 414-420. 


\section{Im Text zitierte Literatur}

(An biographischen Würdigungen sind nur die von Abderhalden verfaßten berücksichtigt.)

${ }^{1}$ Emil Abderhalden, Über den Einfluß des Höhenklimas auf die Zusammensetzung des Blutes. Z. Biol.43 (1902) 125-194 (Diss. med. Basel 1902).

${ }^{2}$ Gustav von Bunge. Med.Klin.16 (1920) $1247 \mathrm{f}$.

${ }^{3}$ Gustav von Bunge. Schweiz. med. Wschr. 50 (1920) 1192-1194.

${ }^{4}$ Zum 100. Geburtstag Gustav von Bunges. Basler Nachrichten, Nr. 26, 18. Januar 1944.

${ }^{5}$ Emil Fischer. Münch.med.Wschr.66 (1919) 938-940.

${ }^{6}$ Emil Fischer †. Med.Klin.15 (1919) $756 \mathrm{f}$.

${ }^{7}$ Die Bedeutung von Emil Fischers Lebenswerk für die Physiologie und darüber hinaus für die gesamte Medizin. Naturwiss. 7 (1919) 860-868.

${ }^{8}$ Schutzfermente des tierischen Organismus, Berlin (Springer) 1912. (2.-4. Aufl.: Abwehrfermente des tierischen Organismus, Berlin 1913-14.)

${ }^{9}$ Familiäre Cystindiathese. Z.physiol. Chem. 38 (1903) 557-561.

${ }^{10}$ G. Fanconi, Weitere Beiträge zur Cystinkrankheit (Der Amindiabetes). Helv. Paediatr. Acta 1 (1945) 183-205.

11 G. Uschmann, Zeittafel zur Akademiegeschichte. Acta historica Leopoldina, Suppl. I (1977) 93-103.

1250 Jahre Fußball-Club Basel 1893-1943 (Red.Dr. J.Düblin). Eigendruck, Basel 1943.

13 Persönl. Mitt. von J. Düblin 1980.

${ }^{14}$ K. Wezler, Schriftenverzeichnis Emil Abderhalden. Akad. d. Wiss. u. d. Literatur Mainz, Jahrbuch 1951, S. 163-224.

${ }^{15}$ J.C.Poggendorff, Biographisch-literarisches Handwörterbuch der exakten Naturwissenschaften, Bd. VI (Verlag Chemie GmbH), Berlin 1936; Bd.VIIa (Akademie-Verlag), Berlin 1956.

${ }^{16}$ Deutsches Bücherverzeichnis, 23.Bd. 1941-1950, Leipzig (Verlag Börsenverein der deutschen Buchhändler) 1952.

\section{Summary}

A diary is presented of which no scientific use has been made hitherto. In it, the Swiss biochemist, Emil Abderhalden, gives an account of his last years when he had to leave Halle/Germany (1945), where he had lived and worked for most of his life. The diary also informs about the end of his academic career in Zurich.- In addition, the references of articles which Abderhalden published in those years (1944-1950) are listed.

Dr. med. vet. Werner Sackmann

Sieglinweg 10

CH-4125 Riehen 